\title{
Concepts and Architecture for a Thumb-Sized Smart IoT Ultrasound Measurement System
}

\author{
(Invited Paper) \\ Jerker Delsing, Jan van Deventer, Jens Eliasson, Jonny Johansson, Torbjörn Löfquist, Fredrik Sandin, \\ Luleå University of Technology \\ division of EISLAB \\ Luleå, Sweden \\ jerker.delsing@ltu.se
}

\begin{abstract}
This paper presents the technology concepts for a "thumb"-sized self-contained ultrasonic IoT measurement system. An overall architecture is proposed, and key elements are discussed with solutions using existing technology, thus arguing that realization is possible with the current technology.

Such an ultrasonic IoT measurement system is constrained by its size and available energy, although it requires at least decent computational and communication resources. Because streaming data from such a device is not advisable from an energy viewpoint, there is a need for resource efficient (energy, memory and computational power) data analysis.

An architecture with the following parts as well as some implementation details and performance data are proposed here:

- Energy supply, battery and super capacitor

- Transducer excitation achieving almost zero electrical losses

- Event detection sensor interface

- Data aggregation using sparse approximation and learned feature dictionaries, adapted to resource constrained embedded systems

- IoT communication protocols and implementations enabling event -based communication and System of Systems integration capabilities
\end{abstract}

The optimization of system level performance requires each subsystem to be optimized for the specific measurement situation taking into account the subsystem interdependencies. This can be performed using a combined electrical and acoustical model of the system. Here, the model allowing electronic and acoustic co-simulation using SPICE is an important tool bridging the electronic and acoustic domains.

\section{INTRODUCTION}

This paper addresses the combination of technology and knowledge enabling the design and construction of a selfcontained smart thumb-sized ultrasound measurement system with a wireless Internet interface - the Smart IoT Ultrasound Measurement System. The involved technologies and capabilities are

- Ultrasound transducer technology and modeling

- Electronics interfaces, transducer excitation and signal reception

- Internet interface

- Data to information transformation based on machine learning

- Power supply

- Power management

- Electronics integration and production technology
Considering the targeted device size, a human thumb, and the complexity of the measurement task, e.g., ultrasound NDT, or acoustic emission for condition-based maintenance, the following major requirements are plausible:

- Size $<5.3 \mathrm{~cm}^{2}$, cylinder with a diameter of $15 \mathrm{~mm}$ and a height of $30 \mathrm{~mm}$

- US frequency ranges of interest $0.1-50 \mathrm{MHz}$ (parts of, for a single application)

- Communication range: $1-500 \mathrm{~m}$

- Power lifetime $>1$ working day, preferable months; energy consumption $<100 \mu W$; on-board power $>$ $100 \mathrm{mWh}$.

- Integration to higher systems by SOA, e.g., Arrowhead Framework [1]

$\cdot$

Based on the above requirements, it is possible to devise a system architecture using technology that already exists or that can be expected to exist within a few years.

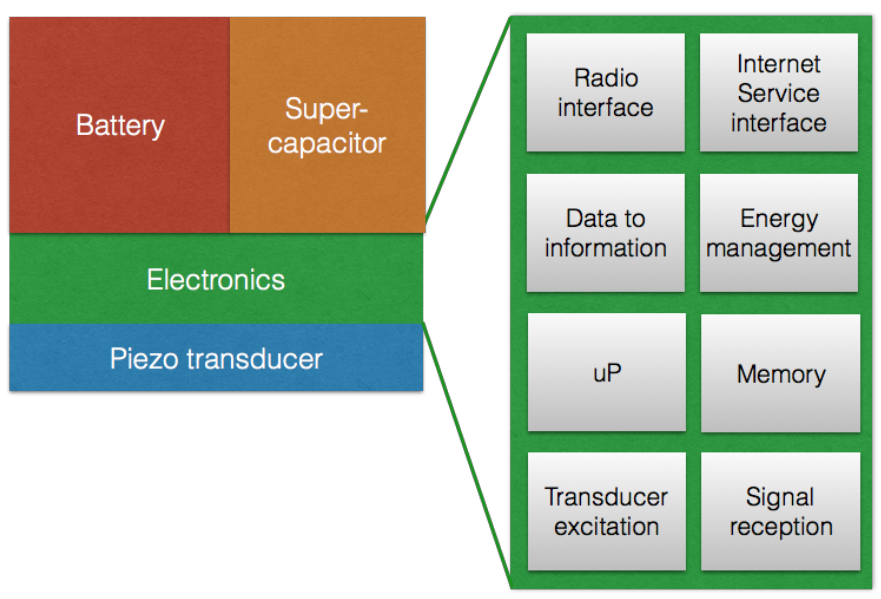

Fig. 1. Main functional blocks of the system architecture for a self-contained IoT ultrasound measurement system.

\section{SySTEM ARCHITECTURE}

The architecture of the IoT US system is depicted in Figure 1. The architecture has a number of major building blocks. As shown, the architecture integrates piezoceramics, electronics 
and a power supply. For the electronics and the power supply, the general requirements imposes some solution complexity.

The power supply is divided into battery and supercapacitor. The electronics are divided in into eight blocks of which 5 are regarded as hardware and 3 are regarded as software dominated.

For all architecture blocks, the energy consumption has to be considered. Managing the energy consumption within each block and between blocks is a major challenge in the system design. Here, the energy management block and its strategies for system and partial system sleep are important.

To address the energy issue and the size limitations, some new and innovative electronics building technologies are explored. A considerable amount of work exists on low-powered sensor nodes, see, for example, [2]-[9]. Some specific examples are schemes handling the reduction of communication, see, e.g., [10], effective routing and multihop schemes, and the reactive partial waking up of WSN and IoT nodes, see, e.g., [11].

Most often, the sensing element itself is disregarded from an energy budget point of view. For the sensor interface, the sensor signal is converted to a digital form, thus enabling detection of numerous signal features through the usage of advanced signal analysis. The most common approach is using an A/D converter. Other well-known approaches involves letting the sensor data influence a digital pulse train of which we easily can measure parameters like frequency, pulse width or duty cycle. None of these approaches put the power consumption of the sensor into focus [12] because it is assumed that energy is not a limiting factor and that all data should be transferred to some computational stage at the WSN/IoT node or any device higher up in the system architecture.

Each of the architecture blocks of the "thumb"-sized US measurement system is discussed below in more detail. Specific technologies necessary to implement block features are presented together with known limitations and energy considerations. The energy considerations and comparisons for all proposed implementations of the different architectural blocks are given in Section III-A.

\section{A. Transducer excitation}

Many ultrasound measurement applications are active, where sound is introduced to the "specimen" under investigation. The response is received and analyzed. Typical examples are transit time flow measurements, where the sound transit time is measured between a transmitter and a receiver, and NDT, where reflected sound bursts are received and analyzed. In such active ultrasound systems, the excitation of a piezoelectric disc is a necessity.

Thus, the objective of this block is to create the sound pressure amplitudes necessary using the least energy, in other words, to minimize energy losses in the excitation circuitry.

It has been shown that the lead inductance from the circuitry to the transducer plays a key role in energy dissipation [13]. Additional losses comes from parasitic capacitances and inductances [14]. The minimization of these losses can only be achieved by shortening lead lengths and minimizing interconnect dimensions in combination with a circuit design that utilizes the energy storage provided by the piezoelectric transducer [15]. It is obvious that an ASIC implementation of such a circuit design is desirable. Innovative placements of the electronics circuitry and the usage of non-conventional production technologies are next. [13] showed that the piezoelectric disc could be used as a multichip module substrate, cf. Figure 2. Here, classical wire bonding was used, reducing the lead length to a few $100 \mu m$. Bonding can be replaced by sequential build up technologies, implementing direct copper interconnects from the ASIC pad to the piezo material metallization [16]. In this way, the interconnect length can be reduced to less than $100 \mu \mathrm{m}$.

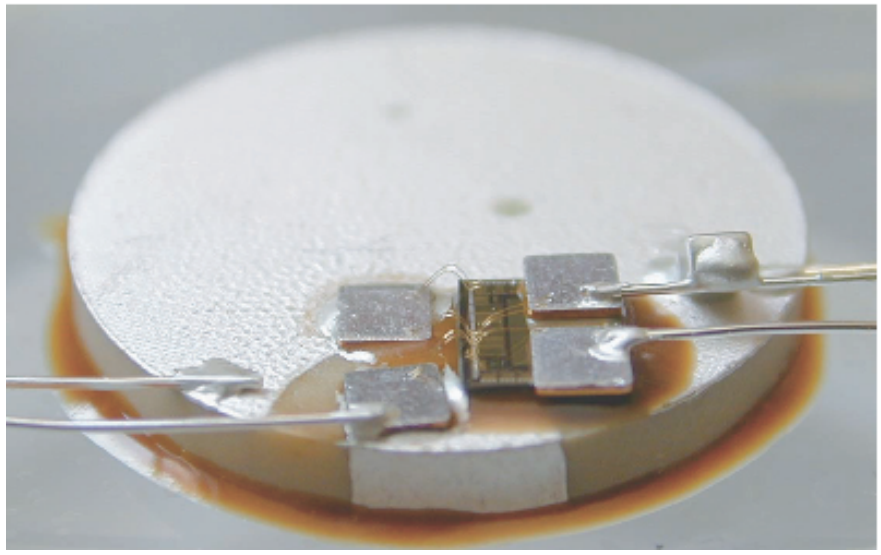

Fig. 2. Photograph of an excitation ASIC circuit mounted system architecture (main functional blocks) of a self contained IoT ultrasound measurements system.

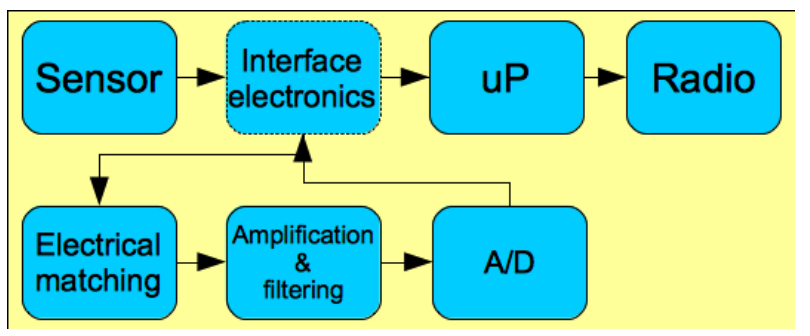

Fig. 3. Traditional WSN/IoT node architecture, A1. All architectural blocks are implemented at the WSN/IoT node.

\section{B. Low power analog front end architecture}

The most frequently used approach is to read the data into the $\mu \mathrm{P}$ store and compare it with the previous data. Energy is spent on sensing element sampling, signal amplification and filtering, A/D conversion and data comparison in the $\mu \mathrm{P}$, cf. Figure 3. This is the architecture found in most WSN and IoT (Internet of Things) nodes currently available, see, e.g., [17], [18].

Using an event-based approach, it is possible to keep larger parts of the electronics in sleep mode for longer times. Based on a previous work [2], three different architectures targeting 
the early detection of useful data will be described and discussed from an energy usage perspective. As a base line, the generic architecture in Figure 3 will be used.

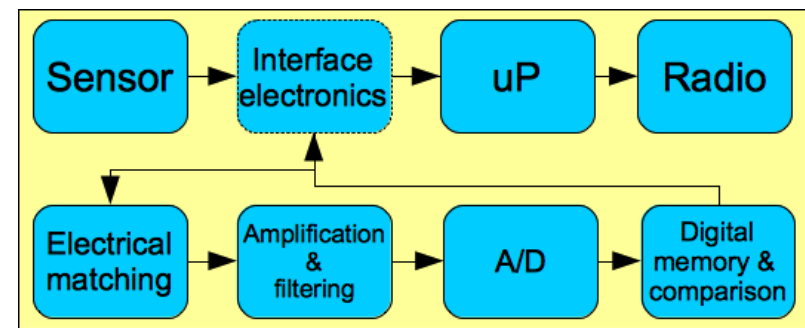

Fig. 4. WSN/IoT node architecture, A2, with external digital sensor memory and comparison logic.

The first design for comparison is by adding a digital memory and comparison function after the A/D conversion, as shown in Figure 4. Energy is spent on sensor sampling, signal amplification and filtering, A/D conversion and storage and comparison of the data in digital memory. Enabling the $\mu \mathrm{P}$ to sleep while performing sensor data sampling if there are no changes or small changes in sensor data will use energy only for the HW cost of digital memory, some configurable logic (to allow for the setting of change limits) and sending a wake-up signal to the $\mu \mathrm{P}$.

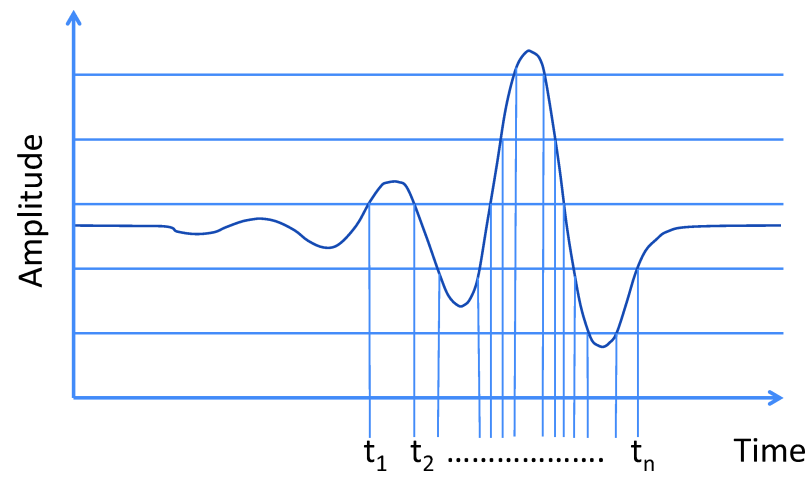

Fig. 5. Illustration of level trigger sampling of a periodic signal.

However, another design is to replace the conventional A/D converter in the above structures with an Asynchronous, or Level-Crossing, ADC. As introduced in [19], [20], the level crossing ADC samples the signal when it passes a predetermined threshold level, instead of at discrete time intervals as performed in traditional ADCs. Thus, the main output of the level-crossing ADC is the time elapsed as the signal has moved between two pre-determined levels, Figure 5. This architecture offers a couple of advantages over traditional techniques. First, it allows the use of fewer quantization levels, as long as the time measurement can be performed with sufficient accuracy. Second and, in the context of this paper, more importantly, the activity of the device is controlled by the behavior of the signal that is to be sampled, allowing event-driven data acquisition [21]. In the sensing scenario, the device will inherently remain idle as long as the signal stays between two threshold levels.
Accordingly, no data are fed through to higher levels in the system, allowing these to remain idle. These possibilities in the design of sensor front ends have been explored for ultrasound measurement systems [22] as well as for other energy constrained sensor applications [23].

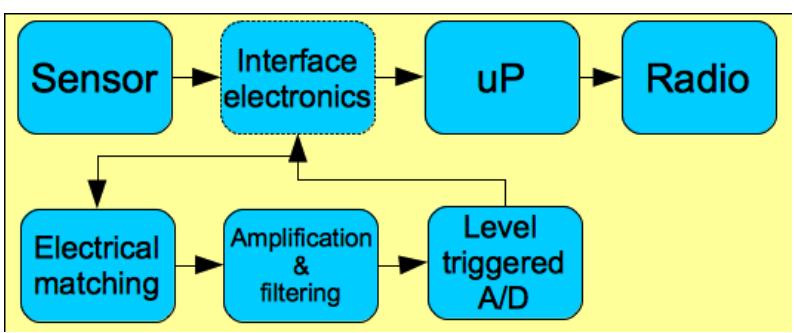

Fig. 6. WSN node architecture, A3, with level triggered A/D conversion.

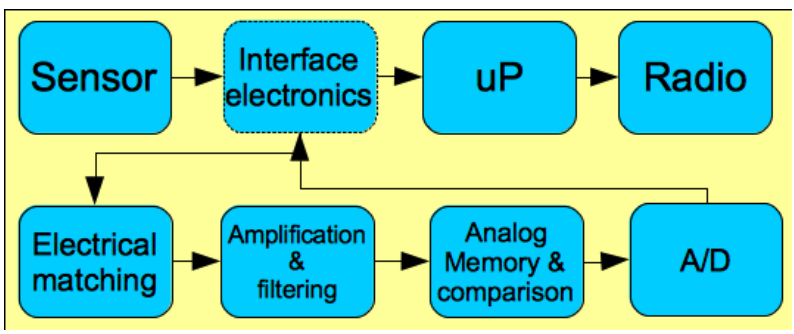

Fig. 7. WSN node architecture, A4, with external analog memory and comparison logic before the A/D conversion.

In terms of energy cost, the $\mathrm{A} / \mathrm{D}$ conversion is dominant in the scenarios discussed above. Thus, it is of interest to explore the possibilities to completely remove the A/D converter. One scenario to achieve this is shown in Figure 6. Here, we introduce an analog memory with the capability of storing and comparing at least two values with associated logic and signaling to the $\mu \mathrm{P}$. In this setup, energy is spent on sensor sampling, signal amplification and filtering, and analog storage with its associated logic. Thus, the $\mu \mathrm{P}$ can sleep during sensing element data sampling if there are no or only small changes in the sensed data.

Two ways to implement the analog memory are the use of charge couple devices (CCD) and switched capacitor storage. CCDs operate by transferring stored charge packets along the surface of a semiconductor by manipulating the potentials of gate electrodes placed close to the semiconductor surface [24]. This forms essentially an analog shift register. While these devices have most commonly been used as image sensors, a number of works have over the years applied them as analog delay elements in signal processing applications. One critical advantage of using CCDs in analog delay circuits is the fact that as long as they are operated at a constant clock frequency, they are completely insensitive to matching between storage cells. In addition, the unique possibility of non-destructive readout at intermediate locations along the device have given rise to CCD-based analog finite impulse response filters [25]. However, to move the charge packets along the surface of the semiconductor, the potentials at the gates need to change as 
functions of time. The charge required to affect the required change in potential is comparable to the total charge currently being transported through the device. Thus, for a CCD the power dissipation depends not only on the charge packet size required to attain the required SNR but also directly on the number of storage elements.

In contrast to CCDs, the only charge required when storing an analog sample using a switch capacitor analog memory is the charge required for the signal itself and some small charge for driving the sampling switch. The charge will stay on its capacitor without further intervention until it becomes corrupted due to leakage. It can be retrieved independently of any other stored charges on other capacitors in a randomaccess manner if desired. Switched capacitor analog memories are however subject to the mismatch between capacitors. Both CCDs and switched capacitor analog memories present interesting alternatives to direct digitalization in systems if a signal needs to be delayed before digitalization or where it is deemed simpler to sample the signal at a high rate and later digitize the stored signal at a slower rate [26].

\section{IoT hardware}

There are currently a number of IoT nodes available on the market, see, for example, [27], [28]. Most of them are built using components off the shelf (COTS) and originate from wireless sensor network nodes. It is obvious that the ASIC solution might be an alternative provided that the volumes are large enough, e.g., $+10^{7}$. In most cases, a hybrid solution is likely the most feasible solution. In this way, one component can be changed with little effort, providing a fast method for incorporating new, e.g., $\mu P$, radio or $\mathrm{A} / \mathrm{D}$ components.

For computational capacity, a 32-bit $\mu P$, e.g., an ARM Cortex M0 or higher, is strongly recommended. The two main argument for this is the need for computational power for the machine learning algorithms and that the cost difference for a smaller $\mu P$ is marginal.

The selection of radio technology is based on the needed communication distance and the bandwidth required by the application. Here, the energy argument show up again. Both distance and bandwidth usage has an energy penalty. The physical limit is related to Shannon's work [29], which states a lower bound on the energy cost per bit transfered. This should be compared with the cost of computation to reduce the amount of data to be transfered. With current available technology, substantial computation is almost always favorable energy-wise compared to sending data over a wired or wireless communication channel.

An IoT ultrasound measurement system that is capable of on-board data to information analytics will reduce the information that needs to be communicated. The expected final data size is expected to be rather small, i.e., 250 Bytes or smaller JSON encoded date per detected event. This suggests a rather low bandwidth radio technology, such as IEEE 802.15 .4 technology with $256 \mathrm{~kb} / \mathrm{s}$ bandwidth [30]. If higher a bandwidth is required, the next step is the latest Bluetooth technologies providing up to $24 \mathrm{Mb} / \mathrm{s}$ [31].

\section{IoT software}

The IoT hardware requires specific software providing the connection to the Internet. To enable wider interoperability, IoT devices have begun to have software stack functionalities incorporating support for the SOA protocol, compression, data encoding and data semantics.

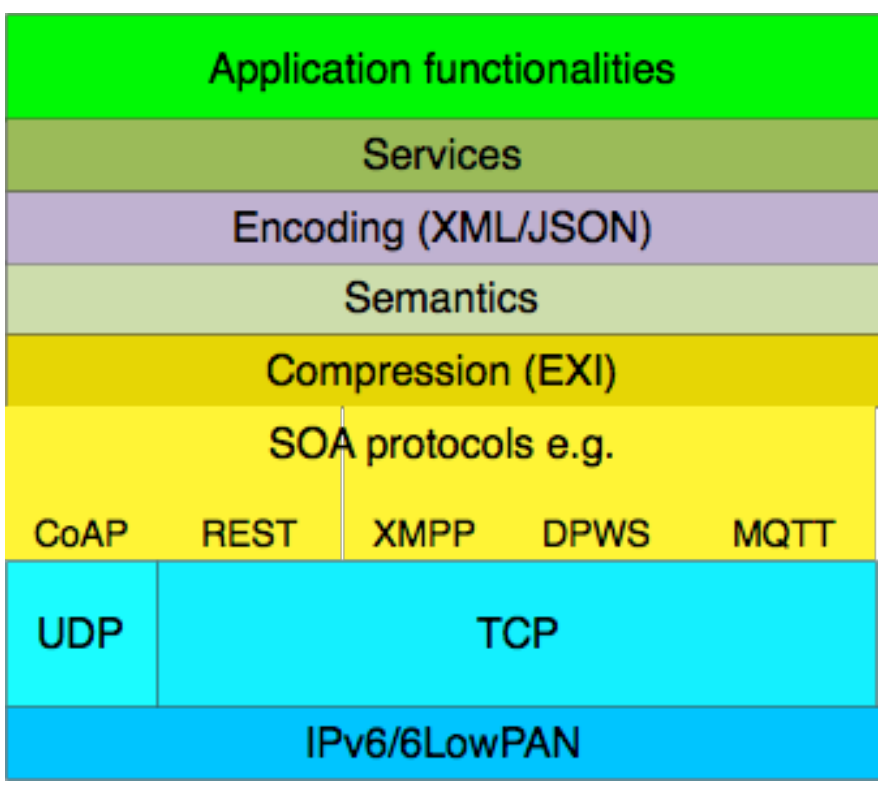

Fig. 8. The main components of the IoT software stack.

An IoT software stack is shown in Figure 8. At the lower levels, standard protocols, such as IPv4/IPv6/6LowPAN plus UDP or TCP, are used [32]-[37]. At this level of an IoT software stack, interoperability across implementations is very high. Here, the data encoding, semantics, concept of services, etc. are left open.

To further enhance interoperability between IoT devices, a considerable amount of work is aimed at the use of serviceoriented architecture, SOA [1], [38], [39]. Here, SOA protocols, encoding, compression and semantics can be integrated to provide the possibility of interoperability at the service level.

There are a number of SOA protocols/architectures developed according to various requirements, e.g., REST, CoAP, XMPP, MQTT [40]-[44]. The data encoding is most often made using XML of JSON [45], [46] and semantics such as SenML and SensML [47], [48]. Compression of the payload occasionally provides significant benefits; here, EXI [49] is the most used solution.

Which combination of software for the software stack is best for the IoT ultrasound measurement system? Given the measurement event nature and constrained resources regarding the computing power, memory, energy supply and communication bandwidth, a suitable choice of technologies would be

- CoAP - SOA protocol for resource constrained devices

- SenML - Light-weight semantics for resource constrained devices

- JSON - Standardized data encoding 


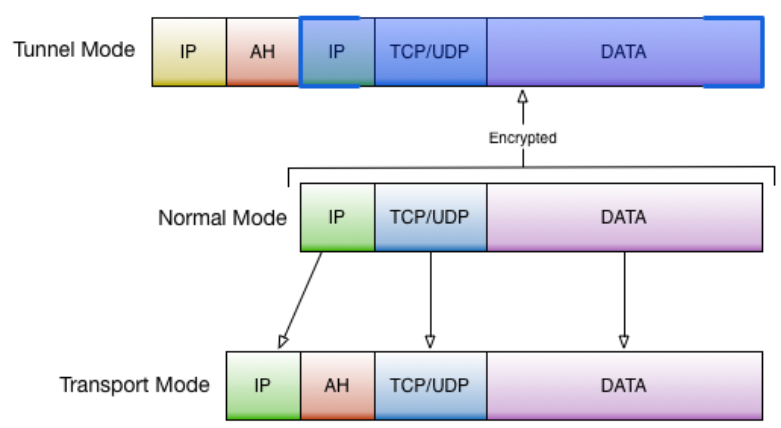

Fig. 9. IPSec encryption and authentication

- EXI - Standardized data compression

The energy cost for the communication of an XML encoded and EXI compressed payload using CoAP protocols is provided by [49].

\section{E. Communication security}

To address the security of the IoT ultrasound measurement system, four aspects have to be considered:

- Data integrity

- Data confidentiality

- Authentication of a service/data consumer

- Authorization of service/data consumption

There are numerous approaches to these security aspects. One of the most interesting ones is IPSec, which is an extension of the IP protocol. IPSec has two different protocols, AH and ESP, to secure the authentication, integrity and confidentiality of communication [50]. IPSec can completely protect the IP datagram (Tunneling Mode [51]) or only the protocols on higher layers (Transport Mode). In Tunneling mode, the IP datagram is encapsulated completely inside a new IP datagram that uses IPSec (the final IP of the datagram could even be different). In Transport mode, IPSec only manages the content of the IP datagram, adding the IPSec header between the original IP header and the header of higher layers, as shown in Figure 9.

To handle the authentication and authorization at the service level, this should be considered in combination with how integration to higher level systems should be implemented. An interesting approach to such integration is provided by the open source Arrowhead Framework [52]. The Arrowhead Framework supports authentication and authorization of service exchanges with two different solutions.

- AA - Authorization Authentication system is defined based on X509 certificates [1], [53].

- AAA - Authorization Authentication Accounting system is defined based on Radius ticket [1], [54], [55].

The AA system is better suited for devices with sufficient computational power. While the AAA system is better suited for resource constrained devices. Thus, in the case of the IoT US system, the AAA solution seems to have advantages. Recent results provide the energy costs for the AAA solution [56].
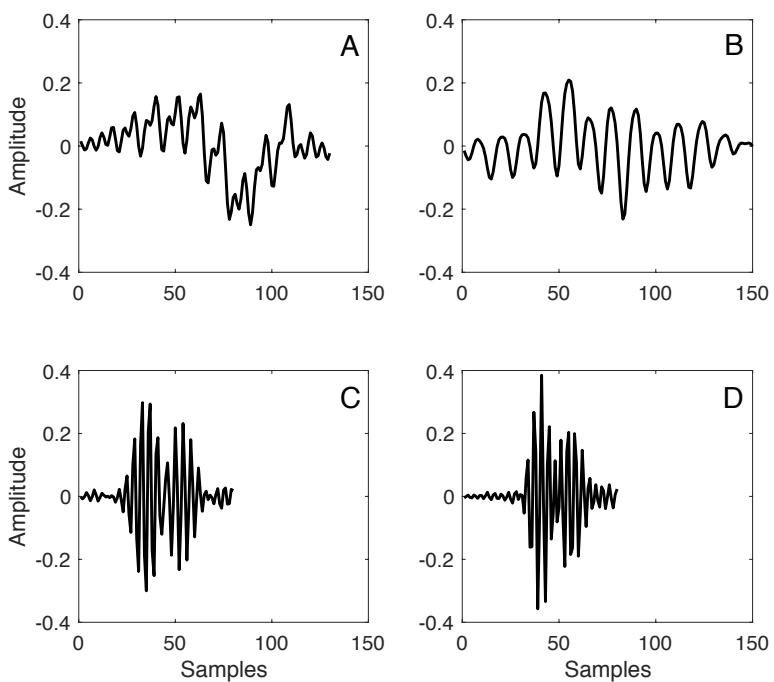

Fig. 10. Four sparse coding atoms learned from acoustic emission from a roller bearing. Atoms A and B are most significant in the no-fault condition, while atoms $\mathrm{C}$ and $\mathrm{D}$ appear when a fault is introduced on the inner race.

\section{F. Data analysis}

Following the event idea, the ambition is to have onboard data analysis, filtering out the events looked for, while using the "thumb"-sized IoT US measurement system. The approach investigated is machine learning and sparse coding with dictionary learning.

Sparse representations of a signal can be calculated if the signal is sparse in some particular transformed space. An ultrasound signal can be approximated by a sparse linear combination of "atoms" from an overcomplete (non-orthogonal) dictionary [57], [58]. Under this condition, an input signal, $\mathbf{x}$, can be represented as

$$
\mathbf{x}=\mathbf{D} \mathbf{s}+\epsilon=\sum_{k=1}^{K} d_{k} s_{k}+\epsilon
$$

where $\mathbf{D}=\left[d_{1}, \ldots, d_{k}\right]$ is a dictionary of atoms $d_{i}$ and $\mathbf{s}$ is the sparse representation of the input signal, $\mathbf{x}$, and $\epsilon$ is an error (noise) term.

There are two major aspects that need to be solved in order to derive such a sparse representation. These aspects are sparse coding and dictionary learning. Sparse coding is the process from which the sparse representation, $\mathbf{s}$, of an input signal is obtained given a known dictionary, D. Dictionary learning is the process that, given some training data, the dictionary $\mathbf{D}$ can be learned. Matching pursuit is one of several algorithms [59] that can be used as part of a sparse coding procedure. There are several algorithms that can be used for dictionary learning, see, for example, [60], [61].

The ultrasound measurement system has been applied to a use case of the acoustic emissions from roller bearings. In Figure 10, sparse coding atoms learned from acoustic emissions from roller bearings are shown. The two atoms at the 


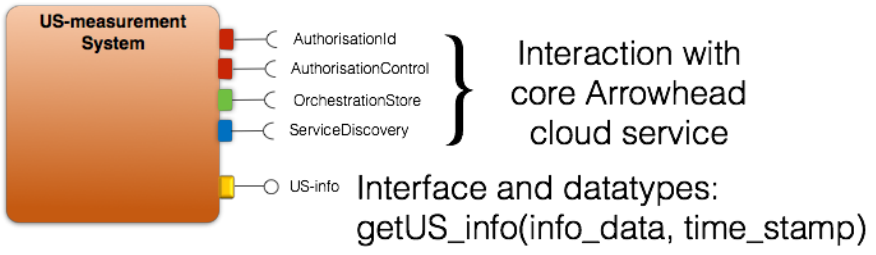

Fig. 11. An Arrowhead Framework service providing processed information from ultrasound pulse echo measurement data.

top (A and B) correspond to a bearing with no faults, meaning that these atoms are essential in the sparse representation of such signals. The two lower atoms (C and D) are learned after a fault condition is introduced in the form of a small defect on the inner race of the rolling element bearing, which means that these atoms are not learned at all for a bearing with no fault. From visual inspection of the evolved atoms, it is clear that the faulty condition changes the dictionary of atoms and consequently also the sparse representation. This enables the detection of a faulty condition. Such detection is currently made using matched filter/FIR filter banks. The detection of changes is less computationally intensive compared to the initial training of atoms which is computationally costly, and therefore possible to implement at resource limited sensor nodes [62], [63]. Thus, initial atom learning can be performed off-line, and the resulting atoms can be downloaded to a resource constrained device such as the "thumb"-sized IoT US measurement system discussed here. The energy costs of the computations indicated above are still to be determined.

\section{G. Measurement data services}

For an IoT US measurement system and any other sensor/measurement system, the ease of integration to higher level systems is important. Being interoperable at a service level seems to provide a simplification regarding integration of any type of IoT device into a System of Systems, SoS. The area of integrating IoT's to a System of Systems is still in an early phase of research. Architecture and technology investigations can be found in [38], [39]. Work extending these including technology implementations has been released as the open source Arrowhead Framework [1], [52].

The investigated and implemented technologies are all based on the service-oriented architecture, SOA. Applying the Arrowhead Framework service concept to the IoT ultrasound measurement system would generate an SOA service as depicted in Figure 11. Here, the service $U S$-info with its interface provides the information created using the machine learning data analysis described above. The service can be subscribed to with an event condition and a check alive time interval.

Depending on the measurement task, the data analysis will give different results. As an application example, the US measurement system is collecting acoustic emissions from a ball bearing. The collected waveforms are processed to provide the Remaining Useful Life, RUL, of the bearing. The RUL together with a time-stamp is provided every time the subscription condition is fulfilled or the keep alive time interval has expired. In this way, the US measurement system can easily be integrated into a larger automation system.

\section{H. System modeling and simulation}

The design of the "thumb-sized" IoT US measurement system requires a number of complex considerations. The use of simulation tools is of great help here. One of the key elements of the "thumb"-sized IoT US measurement system is the tight integration between the piezoceramic transducer material and the electronics. Over the years, advanced circuit models of piezoceramics have been developed [64]-[66]. With these models, it becomes possible to predict the combined electrical, piezoceramic and acoustic behavior of the "thumb"sized IoT US measurement system described above. These models also support prediction of the energy consumption. Thus, a critical parameter given the limited volume for energy storage within the "thumb"-sized IoT US measurement system can be investigated using feasible simulation tools.

\section{SIZE AND ENERGY ESTIMATIONS}

In the following, an energy consumption and size analysis will be made. The energy analysis provides the energy cost for one pulse echo measurements using the electronics described above. Combining this with how much energy can be stored within the given volume gives the number of measurements possible on one charge of the battery.

The energy and size analyses should be regarded as first indications of possibilities. It is further clear that the assumed measurement case is limited compared to the wide possibility of measurement applications using an IoT US measurement system.

\section{A. Energy analysis}

A first energy cost analysis has been performed for an ultrasound pulse echo measurement. The basic measurement specifications are

- US-bandwidth 0.5 - $10 \mathrm{MHz}$

- Sound amplitude resolution: 10 bits

This analysis is conducted using state-of-the-art published data and data from commercial devices. Thus, we can build pictures of the total energy consumptions of the proposed architectures; the energy cost of the data analysis using the machine learning approach is still unknown. Furthermore, no circuit simulations are performed here nor have we built any complete devices.

The energy analysis is based on the following model. The total electrical power $P_{\text {tot }}$ consumed by a WSN node can be described as

$$
\begin{array}{r}
P_{\text {tot }}=P_{\text {sens }}+P_{\text {cond }}+P_{A-m e m}+P_{A D}+P_{D-m e m}+ \\
P_{\mu P}+P_{\text {radio }}
\end{array}
$$

where $P_{\text {sens }}, P_{\text {sens }}, P_{\text {cond }}, P_{A-m e m}, P_{A D}, P_{D-m e m}, P_{\mu P}$, $P_{\text {radio }}$ are the power consumptions for each of the blocks in the compared architectures. These power consumptions are for continuous operation. 
The total energy usage is then obtained by introducing the time needed for each operation (after which it can be turned off). The turn off and turn on times are included in the assumed time for which power is consumed. The power usage time is thus assumed to be the sum of the turn on and off times and the power usage to perform the architectural block action. To make the analysis reasonably simple, we assume that the architecture supports turning off $E_{\text {sens }}$ and $E_{\text {cond }}$, once the data have been stored either in analog or digital form.

For data sampling from one sensor, we assume a sensing and conditioning time $t_{\text {sens }}$ ond , an analog storing time $t_{A-m e m}$, an A/D time $t_{A D}$, a digital memory time $t_{D-m e m}$ and a $\mu \mathrm{P}$ time $t_{\mu P}$. Thus, we obtain the total power $E_{t o t}$ used for data sampling from one sensor as

$$
\begin{aligned}
& E_{\text {tot }}=P_{\text {sens }} * t_{\text {sens }}+P_{\text {cond }} * t_{\text {cond }}+P_{A-m e m} * t_{A-m e m}+ \\
& P_{A D} * t_{A D}+P_{D-m e m} * t_{D-m e m}+P_{\mu P} * t_{\mu P} \\
& P_{\text {radio }} * t_{\text {radio }}
\end{aligned}
$$

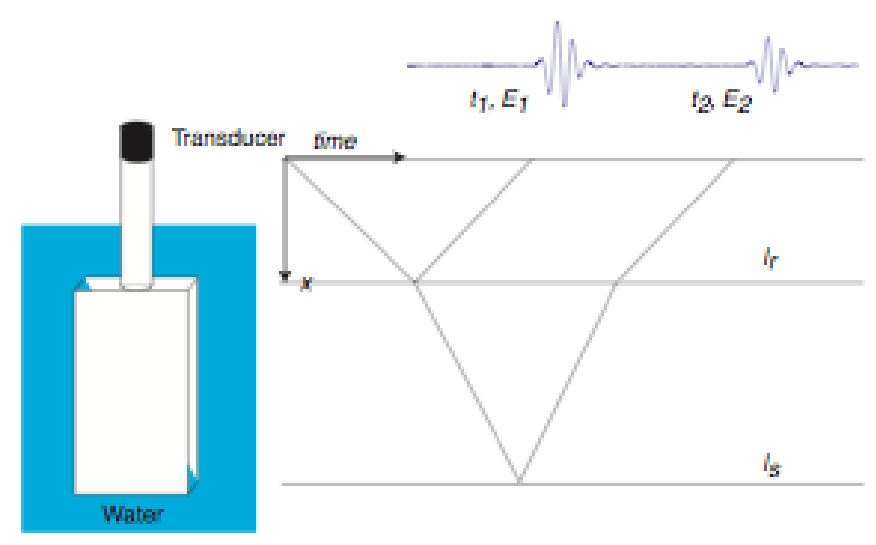

Fig. 12. Ultrasound pulse echo measurement with associated acoustic signals

A typical pulse echo measuring situation with typical sound signals is shown in Figure 12. In Table I, the power consumption and related timing for the sensor and associated electronics are given. Next, the expected span of the maximum and minimum energy consumption is calculated based on the data in Table I. The result is given in Table II.

It is obvious that the signal conditioning, $\mu \mathrm{P}$ and radio use a large amount of energy. Thus, any architecture that can avoid waking up the $\mu \mathrm{P}$ and the radio has clear advantages from an energy consumption point of view. This favors reactive architectures where the $\mu \mathrm{P}$ and the radio remain idle until a valid measurement occurs. Furthermore, integration of advanced signal processing capabilities will play an increasingly important role since the energy needed per computational operation decreases by a factor of two every year and a half [69] and currently reaches about $10^{8}$ FLOPS per mW.

Based on the work in [2], the following general conclusions can be made:
TABLE I

ENERGY CONSUMPTION AND TIMING FOR AN ULTRASOUND WSN SENSOR NODE.

\begin{tabular}{|l|l|l|}
\hline Device & $\begin{array}{l}\text { Energy consump- } \\
\text { tion }\end{array}$ & Time awake $[\mu \mathrm{s}]$ \\
\hline Piezo excitation & $0.01 \mathrm{~mW}[67]$ & $\begin{array}{l}1 \quad(1 \mu \mathrm{s} \mathrm{long} \\
\text { pulse excitation }\end{array}$ \\
\hline Amplifier and filtering & $5 \mathrm{~mW} \mathrm{[68]}$ & cont \\
\hline Analog memory & $0.5 \mu \mathrm{W}$ & cont \\
\hline A/D (10 bit) & $2 \mathrm{~mW}$ & cont \\
\hline level trigged A/D & $175 \mathrm{~mW}$ & cont \\
\hline Digital memory & $0.01-0.1 \mathrm{~mW}$ & 10 (storage time) \\
\hline$\mu \mathrm{P}$ & $1-10 \mathrm{~mW}$ & $\begin{array}{l}30(300 \text { clock cy- } \\
\text { cles at } 10 \mathrm{MHz})\end{array}$ \\
\hline Radio 802.15 .4 & $40-100 \mathrm{~mW}$ & $10 \mathrm{~ms}(250 \mathrm{Bytes})$ \\
\hline
\end{tabular}

TABLE II

LOW- AND HIGH-END ENERGY CONSUMPTION DATA IN W FOR ULTRASOUND PULSE ECHO MEASUREMENT AND THE 4 WSN/IOT NODE ARCHITECTURES A1-A4.

\begin{tabular}{|l|r|r|r|r|}
\hline & $\mathrm{A} 1$ & $\mathrm{~A} 2$ & $\mathrm{~A} 3$ & $\mathrm{~A} 4$ \\
\hline$U S_{\text {low }}$ & $8.4 * 10^{-3}$ & $7.4 * 10^{-3}$ & $2 * 10^{-1}$ & $5.4 * 10^{-3}$ \\
\hline$U S_{\text {high }}$ & $21 * 10^{-3}$ & $8 * 10^{-3}$ & $2 * 10^{-1}$ & $6 * 10^{-3}$ \\
\hline
\end{tabular}

- For low frequency sensor signal

- The $\mu \mathrm{P}$ energy cost dominates

- A/D + digital memory comparison is favorable

- For higher frequency sensors such as ultrasound

- The data conversion energy cost dominates

- Analog memory storage and comparison has the most potential

- A reactive architecture results in an improved energy budget in all cases

Thus, it is clear that in a sensing situation with a dynamic sensor signal, such as ultrasound, avoiding A/D conversion is a promising approach. If analog memory and comparison techniques can be developed similar to what we have seen for $\mathrm{A} / \mathrm{D}$ converters, an analog storage architecture will be a strong contender for future WSN/IoT designs.

\section{SizE ESTIMATION}

Determining the size limit of a "thumb-sized" IoT US measurement system requires size estimates of the major architectural blocks according to Figure 1:

- Battery

- Super capacitor

- Electronics

- Piezoceramic plate

Start from the piezoceramic plate and assume a "thumb" diameter of $15 \mathrm{~mm}$. Assuming $2 \mathrm{~mm}$ of encapsulation gives a piezo disk with an $11 \mathrm{~mm}$ diameter. Assuming a $1 \mathrm{MHz}$ transducer center frequency and some PZ material gives a thickness of $2 \mathrm{~mm}$.

The electronics circuits then have a maximum diameter of $11 \mathrm{~mm}$. Here, the following electronics blocks should be accommodated:

- Transducer excitation 
First layer of components with solder less interconnects
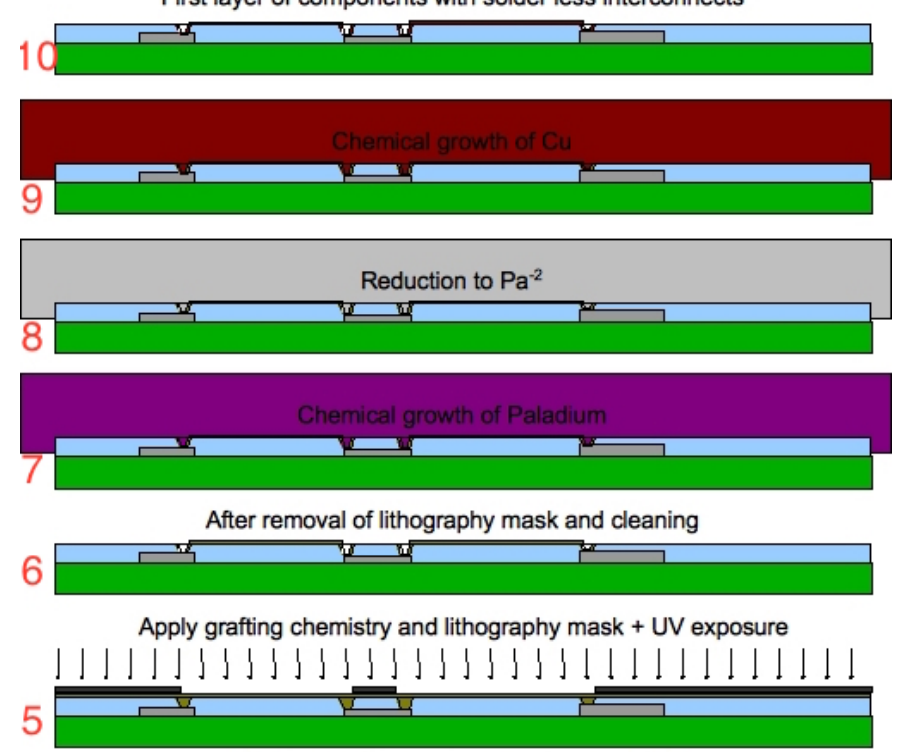

Drilling of via to components and conductors

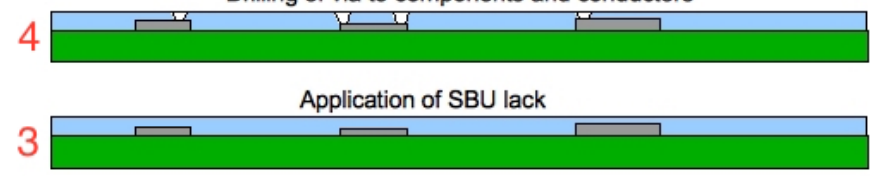

Placement of components, chip and passivs

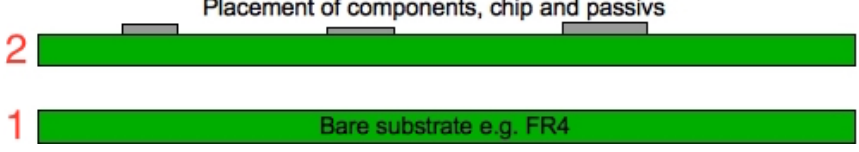

Fig. 13. A side view of the IoT electronics indicating the Sequential Build Up production process. For a second layer of components, the process is repeated from step 2.

- Signal reception

- $\mu P$

- Memory

- Radio interface

- Necessary passive components

According to Section II-A, the excitation electronics should have as short leads as possible to the piezoceramic disk. Thus, using the ceramic disk as an electronic substrate is advisable. To reduce the disturbances from the radio and the $\mu P$, it is advisable to mount the signal reception electronics at the piezoceramic.

Provided the use of advanced production technologies, the required interconnects between components should be possible to make using, e.g., sequential build up [70], [71] of direct copper interconnects.

The available substrate area, $2 * \pi * r^{2}$, becomes a maximum of $200 \mathrm{~mm}^{2}$. Assuming maximum $\mu P$, radio, memory excitation and amplifier sizes of $50 \mathrm{~mm}^{2}$ indicates that two layers of electronics are necessary. Thus, a side view of the electronics may look like, Figure 13. Assuming a maximum of $1 \mathrm{~mm}$ for each layer adds $2 \mathrm{~mm}$ of electronics.

This will leave the rest of the "thumb" space for a battery and a super-capacitor. The supercapacitor should provide the energy and high current for the communication over the radio link.

Assuming a radio transmission power consumption of 20 $\mathrm{mW}$ and a transmission time of approximately $10 \mathrm{~ms}(250$ Bytes over a $250 \mathrm{~kb} / \mathrm{s}$ radio link) results in a high current energy requirements of some $0.05 \mu W h$. With an energy density of $5 W h / L[72]$ and a supercapacitor diameter of 11 $\mathrm{mm}$, a thickness of a $1 \mathrm{~mm}$ would provide enough high current power storage for about 3 transmissions of 250 Bytes. The super capacitor is recharged from the battery.

For the battery, we assume the use of some type of lithiumion based technology. Here, the energy per volume is in the ballpark of $300 W h / L$ [73]. Assuming a battery height of $20 \mathrm{~mm}$ over the $11 \mathrm{~mm}$ diameter provides more than $1.500 \mathrm{Ws}$ of energy. This would allow more than 2.000 ultrasound measurements including wireless data transfer for each measurement. Enabling a longer "thumb" will of course allow for more measurements.

It should be noted here that the above energy calculations do not include the machine learning computations discussed in Section II-F.

\section{Conclusion}

An architecture for a "thumb" IOT US measurement system is proposed. Architecture details addressing both passive and active ultrasound measurements and their low power operation are provided. The measurement system will be wirelessly connected to the Internet with the ability to provide a service interface enabling integration with higher level systems. Thus, the "thumb"-sized IoT measurement system described here can be regarded as an IoT device that is capable of being integrated into a System of Systems cloud.

The production of a "thumb" US system seems to be technically feasible considering currently known production technologies.

Energy consumption data or estimates for most architectural blocks indicates that the "thumb" size ultrasound measurement system will be capable of more than 2.000 pulse echo measurements. This will be sufficient for a single day or more days of operation depending on the application.

\section{REFERENCES}

[1] e. J Delsing, Ed., IoT Automation - Arrowhead Framework. CRC Press, Taylor and Francies, To appear fall 2016, 2016.

[2] J. Delsing, J. Borg, and J. Johansson, "New architecture for efficient data sampling in wireless sensor network devices," 2013.

[3] W. Heinzelman, A. Chandrakasan, and H. Balakrishnan, "Energyefficient communication protocol for wireless sensor networks," in Proc. Hawaii Intl. Conf. System Sciences, Hawaii, Jan 4-7 2000, pp. 30053014.

[4] J. Eliasson, M. Lundberg, and P. Lindgren, "Time synchronous bluetooth sensor network," in Proc- IEEE Concumer Communication and Networking Conference, CCNC, 2006.

[5] M. Lundberg, J. Eliasson, L. Svensson, and P. Lindgren, "Context aware power optimization of wireless embedded internet system," in Proceedings IEEE IMTC, 2004.

[6] J. Eliasson, P. Lindgren, J. Delsing, S. J. Thompson, and Y.-B. Chen, "A power management architecture for wireless sensor nodes," in Proc IEEE Wireless Communication and Networking Conference, WCNC, 2007. 
[7] V. Loscri, G. Morabito, and S. Marano, "A two level hierarchy for low energy adaptive clustering hierarchy (TL-LEACH)," in Proc. 62nd IEEE Vehicular Technology Confere)nce (VTC-Fall, Dallas, 25-28 September 2008, pp. 1809-1813.

[8] Y. Xu, J. Heidman, and D. Estrin, "Geography-informed energy conservation for ad hoc routing," in Proc. Mobicom, 2001, pp. 70-84.

[9] J. Delsing and P. Lindgren, "Sensor communication technology towards ambient intelligence, a review," Meas. Sci. Technol., vol. 16, pp. 37-46, 2005.

[10] J. Lu, F. Valois, M. Dohler, and M.-Y. Wu, "Optimized data aggregation in wsns using adaptive arma," in Proceeedings Sensorcomm 2010, 2010, pp. $115-120$.

[11] S. G. Hong, N. S. Kim, C. S. Pyo, and W. W. Kim, "Hybrid sensor module and data processing using low-power wakeup in wsn," in Proceeedings Sensorcomm 2010, 2010, pp. 191-195.

[12] M. Vieira, C. Coelho, J. da Silva, D.C., and J. da Mata, "Survey on wireless sensor network devices," in Emerging Technologies and Factory Automation, 2003. Proceedings. ETFA '03. IEEE Conference, vol. 1, Sept 2003, pp. 537-544 vol.1.

[13] J. Johansson and J. Delsing, "A compact ultrasonic transducer using the active piezoceramic material as electronics carrier," in Proc IMAPS workshop. Denver: IMAPS, April 2004.

[14] —, "Effects of parasitic electrical components on an ultrasound system: measurements and simulations using spice models," in Proc. of the SPIE - International Society for Optical Engineering, vol. 4946. SPIE, 2003, pp. 174-182.

[15] J. Borg, J. Johansson, J. Van Deventer, and J. Delsing, Reciprocal operation of ultrasonic transducers: experimental results, ser. I E E E International Ultrasonics Symposium. Proceedings. IEEE Communications Society, 2006, pp. $1013-1016$.

[16] J. Fjelstad, "Solderless assembly of electronic products - a more reliable and more cost effective approach to electronics manufacturing?" in Proceedings IEEE Vehicle Power and Propulsion Conference, VPPC '09, 2009, pp. 11-16, cited By (since 1996) 0. [Online]. Available: http://www.scopus.com/inward/record.url?eid=2-s2.0-72149113016 \&partnerID $=40 \backslash \& \mathrm{md} 5=4 \mathrm{~d} 0280 \mathrm{a} 85041$ ea0e4c6a637f8996149b

[17] EISTEC. (2013) Eistec. [Online]. Available: http://www.eistec.se

[18] Memsic. (2011, Dec) Wireless modules. [Online]. Available: http: //www.memsic.com/wireless- sensor-networks/

[19] N. Sayiner, H. Sorensen, and T. Viswanathan, "A level-crossing sampling scheme for a/d conversion," Circuits and Systems II: Analog and Digital Signal Processing, IEEE Transactions on, vol. 43, no. 4, pp. 335 -339, apr 1996.

[20] E. Allier, G. Sicard, L. Fesquet, and M. Renaudin, "A new class of asynchronous a/d converters based on time quantization," in Asynchronous Circuits and Systems, 2003. Proceedings. Ninth International Symposium on, May 2003, pp. 196 - 205.

[21] Y. Tsividis, "Event-driven data acquisition and digital signal processing a tutorial," Circuits and Systems II: Express Briefs, IEEE Transactions on, vol. 57, no. 8, pp. $577-581$, aug. 2010.

[22] K. Kozmin, J. Johansson, and J. Delsing, "Level-crossing adc performance evaluation toward ultrasound application," Circuits and Systems I: Regular Papers, IEEE Transactions on, vol. 56, no. 8, pp. 1708 -1719, aug. 2009.

[23] M. Trakimas and S. Sonkusale, "An adaptive resolution asynchronous adc architecture for data compression in energy constrained sensing applications," Circuits and Systems I: Regular Papers, IEEE Transactions on, vol. 58, no. 5, pp. $921-934$, may 2011.

[24] R. Baertsch, W. Engeler, I. Goldberg, H.S., C. Puckette, and J. Tiemann, "The design and operation of practical charge-transfer transversal filters," Solid-State Circuits, IEEE Journal of, vol. 11, no. 1, pp. 65 - 74, feb 1976.

[25] D. D. Buss, D. R. Collins, W. H. Bailey, and C. R. Reeves, "Transversal filtering using charge-transfer devices," Solid-State Circuits, IEEE Journal of, vol. 8, no. 2, pp. 138-146, 1973.

[26] G. Haller and B. Wooley, "A 700-mhz switched-capacitor analog waveform sampling circuit," Solid-State Circuits, IEEE Journal of, vol. 29, no. 4, pp. $500-508$, apr 1994

[27] J. Johansson, M. Völker, J. Eliasson, Å. Östmark, P. Lindgren, and J. Delsing, "MULLE:a minimal sensor networking device - implementation and manufacturing challenges," in Proc. IMAPS Nordic. IMAPS, 2004.

[28] "Wsn platforms," http://wsn.oversigma.com/wiki/index.php?title=WSN Platforms.
[29] C. E. Shannon, "A mathematical theory of communication," The Bell System Technical Journal, vol. 27, no. 4, pp. 623-656, Oct 1948.

[30] J. P. K. Gilb and et.al., "Ieee standard for local and metropolitan area networks - part 15.4: Low-rate wireless personal area networks (lrwpans)," IEEE, Standard, 2011.

[31] Wikipedia, "Bluetooth - wikipedia, the free encyclopedia," 2016, [Online; accessed 3-July-2016]. [Online]. Available: https://en.wikipedia.org/w/index.php?title=Bluetooth\&oldid=727899104

[32] —, "User datagram protocol - wikipedia, the free encyclopedia," 2016, [Online; accessed 20-April-2016]. [Online]. Available: https://en.wikipedia.org/w/index.php?title=User_Datagram_ Protocol\&oldid $=715583050$

[33] J. Postel, "Rfc 768 user datagram protocol," IETF, Tech. Rep., 1980.

[34] V. Cerf and R. Kahn, "A protocol for packet network intercommunication," IEEE Transactions on Communications, vol. 22, no. 5, pp. 637648, May 1974.

[35] Wikipedia, "Transmission control protocol - wikipedia, the free encyclopedia," 2016, [Online; accessed 7-July-2016]. [Online]. Available: https://en.wikipedia.org/w/index.php?title=Transmission_Control_ Protocol\&oldid $=728151498$

[36] M.Wasserman, Recomendation for IPv6 in Third Generation Partnership Project (3GPP) standard, ISOC Std. RFC 3314, September 2002.

[37] Z. Shelby and C. Bormann, 6LoWPAN: The Wireless Embedded Internet. Wiley, 2009.

[38] A. W. Colombo, T. Bangemann, S. Karnouskos, J. Delsing, P. Stluka, R. Harrison, F. Jammes, and J. L. Lastra, "Industrial cloud-based cyberphysical systems," The IMC-AESOP Approach, 2014.

[39] F. Jammes, B. Bony, P. Nappey, A. W. Colombo, J. Delsing, J. Eliasson, R. Kyusakov, S. Karnouskos, P. Stluka, and M. Till, "Technologies for soa-based distributed large scale process monitoring and control systems," in IECON 2012-38th Annual Conference on IEEE Industrial Electronics Society. IEEE, 2012, pp. 5799-5804.

[40] C. Pautasso, E. Wilde, and R. Alarcon, Eds., REST: Advanced Research Topics and Practical Applications. Springer, 2014.

[41] Z. Shelby, "The constrained application protocol (coap) - rfc 7252," IETF, Tech. Rep., 2014.

[42] (2016) Xmpp is the open standard for messaging and presence. [Online]. Available: http://xmpp.org

[43] D. Driscoll and A. Mensch, "Oasis devices profile for web services (dpws) version 1.1," OASIS Tech. Rep., 2009. [Online]. Available: http://docs. oasis-open.org/ws-dd/dpws/1.1/os/wsdd-dpws-1.1-spec-os.pdfhttp: //docs.oasis-open.org/ws-dd/dpws/1.1/os/wsdd-dpws-1.1-spec-os.pdf

[44] (2016) Mqtt is a machine-to-machine ( $2 \mathrm{~m})$ /"internet of things" connectivity protocol. [Online]. Available: http://mqtt.org

[45] (2016) Extensible markup language - xml. [Online]. Available: https://en.wikipedia.org/wiki/XML

[46] (2016) Introducing json. [Online]. Available: http://www.json.org

[47] C. Jennings and Z. Shelby, "Media types for sensor markup language (senml) draft-jennings-senml-10," IETF, Tech. Rep., 2013.

[48] M. Botts and A. Robin. (2014) Ogc sensorml: Model and xml encoding standard.

[49] R. Kyusakov, P. Punal, J. Eliasson, and J. Delsing, "Exip: A framework for embedded web development," ACM Transactions on the Web, vol. 8, no. 4, 2014

[50] V. Manral, "Cryptographic Algorithm Implementation Requirements for Encapsulating Security Payload (ESP) and Authentication Header (AH)," RFC 4835 (Proposed Standard), Internet Engineering Task Force, Apr. 2007. [Online]. Available: http://www.ietf.org/rfc/rfc4835.txt

[51] S. Kent, "IP Encapsulating Security Payload (ESP)," RFC 4303 (Proposed Standard), Internet Engineering Task Force, dec 2005. [Online]. Available: http://www.ietf.org/rfc/rfc4303.txt

[52] (2016) Arrowhead framework wiki. [Online]. Available: https://forge. soa4d.org/plugins/mediawiki/wiki/arrowhead-f/index.php/Main_Page

[53] M. Myers, R. Ankney, A. Malpani, S. Galperin, and C. Adams, "X. 509 internet public key infrastructure online certificate status protocol-ocsp," RFC 2560, Tech. Rep., 1999.

[54] A. DeKok and A. Lior, "Remote authentication dial in user service (radius) protocol extensions," RFC 6929, April, Tech. Rep., 2013.

[55] P. Punal, J. Eliasson, and J. Delsing, An Authentication and Access Control Framework for CoAP-based Internet of Things. IEEE, 2015, pp. 5293-5299.

[56] P. P. Pereira, J. Eliasson, and J. Delsing, "Efficient framework for industrial iot," IEEE INTERNET OF THINGS JOURNAL, 2016. 
[57] A. M. Bruckstein, D. L. Donoho, and M. Elad, "From sparse solutions of systems of equations to sparse modeling of signals and images," SIAM Review, vol. 51, no. 1, pp. 34-81, 2009.

[58] Y. Li, Sparse representation for machine learning, ser. Advances in Artificial Intelligence, Lecture Notes in Computer Science. Springer, 2013, vol. 7884, pp. 352-357.

[59] H. Lee, A. Battle, R. Raina, and A. Ng, "Efficient sparse coding algorithms," in Advances in neural information processing systems, 2006, pp. 801-808.

[60] K. Kreutz-Delgado, J. F. Murray, B. D. Rao, K. Engan, T.-W. Lee, and T. J. Sejnowski, "Dictionary learning algorithms for sparse representation," Neural Computation, vol. 15, no. 2, pp. 349-396, 2003.

[61] E. C. Smith and M. S. Lewicki, "Efficient auditory coding," Nature, vol. 439, pp. 978-981, Feb. 2006.

[62] J. N. J. E. Sergio Martin del Campo, Kim Albertsson and F. Sandin, "Fpga prototype of machine learning analog-to-feature converter for event-based succinct representation of signals," in IEEE International Workshop on Machine Learning for Signal Processing, Southampton, UK, 2013.

[63] S. M. del Campo and F. Sandin, "Towards zero-configuration condition monitoring based on dictionary learning," in Signal Processing Conference (EUSIPCO), 2015 23rd European, Aug 2015, pp. 1306-1310.

[64] M. Redwood, "Transient performance of a piezoelectric transducer," The Journal of the Acoustical Society of America, vol. 33, no. 4, pp. 527-536, 1961. [Online]. Available: http://scitation.aip.org/content/asa/ journal/jasa/33/4/10.1121/1.1908709

[65] A. Puttmer, P. Hauptmann, R. Lucklum, O. Krause, and B. Henning, "Spice model for lossy piezoceramic transducers," IEEE Transactions on Ultrasonics, Ferroelectrics, and Frequency Control, vol. 44, no. 1, pp. 60-66, Jan 1997.

[66] J. van Deventer, T. Lofqvist, and J. Delsing, "Pspice simulation of ultrasonic systems," IEEE Transactions on Ultrasonics, Ferroelectrics, and Frequency Control, vol. 47, no. 4, pp. 1014-1024, July 2000.

[67] J. Johansson and J. Delsing, "Energy and pulse control possibilities using ultra-tight integration of electronics and piezoelectric ceramics," in Proc. $U F F C$, vol. 3, 2004, pp. 206-2210.

[68] Texas instruments opa683. [Online]. Available: http://www.ti.com/ product/opa683

[69] J. Koomey, S. Berard, M. Sanchez, and H. Wong, "Implications of historical trends in the electrical efficiency of computing," IEEE Annals of the History of Computing, vol. 33, no. 3, pp. 46-54, 2011.

[70] J. Fjelstad, "Reversing the electronic assembly process," Semiconductor International, vol. 32, no. 13, pp. 2428, 2009, cited By (since 1996) 0. [Online]. Available: http://www.scopus.com/inward/record.url?eid=2-s2.0-75749088963 \ \&partnerID $=40 \backslash \&$ md5=8f1ea94ba4761e4b221aff2ac5972933

[71] - "Retrospective on electronics technology and prospective methods for co-design of ic packaging and manufacturing improvements," 2009, pp. 559-564, cited By (since 1996) 0. [Online]. Available: http://www.scopus.com/inward/record.url?eid=2-s2.0-67649664597 \&partnerID $=40 \backslash \& \mathrm{md5}=3 \mathrm{ec} 69 \mathrm{e} 4 \mathrm{aa} 4 \mathrm{a} 160487380 \mathrm{c} 248 \mathrm{~b} 5 \mathrm{beba} 01$

[72] Wikipedia, "Supercapacitor - wikipedia, the free encyclopedia," 2016, [Online; accessed 3-July-2016]. [Online]. Available: https: //en. wikipedia.org/w/index.php?title=Supercapacitor\&oldid=727682118

[73] —, "Comparison of battery types - wikipedia, the free encyclopedia," 2016, [Online; accessed 3-July-2016]. [Online]. Available: https://en.wikipedia.org/w/index.php?title=Comparison_of_ battery_types\&oldid=726089067 